\title{
Trichoderma asperelloides Suppresses Nitric Oxide Generation Elicited by Fusarium oxysporum in Arabidopsis Roots
}

\author{
Kapuganti J. Gupta, ${ }^{1}$ Luis A. J. Mur, ${ }^{2}$ and Yariv Brotman ${ }^{3}$ \\ ${ }^{1}$ Department of Plant Sciences, University of Oxford, South Parks Road, Oxford OX1 3RB, U.K.; ${ }^{2}$ Aberystwyth University, \\ Institute of Biological, Environmental and Rural Science, Edward Llwyd Building, Aberystwyth, SY23 3DA U.K.; ${ }^{3}$ Max- \\ Planck-Institute of Molecular Plant Physiology, Am Mühlenberg 1, D-14476 Golm-Potsdam, Germany
}

Submitted 4 June 2013. Accepted 16 November 2013.

Inoculations with saprophytic fungus Trichoderma spp. are now extensively used both to promote plant growth and to suppress disease development. The underlying mechanisms for both roles have yet to be fully described so that the use of Trichoderma spp. could be optimized. Here, we show that Trichoderma asperelloides effects include the manipulation of host nitric oxide (NO) production. NO was rapidly formed in Arabidopsis roots in response to the soil-borne necrotrophic pathogen Fusarium oxysporum and persisted for about $1 \mathrm{~h}$ but is only transiently produced (approximately $10 \mathrm{~min}$ ) when roots interact with $T$. asperelloides (T203). However, inoculation of $F$. oxysporum-infected roots with $T$. asperelloides suppressed $F$. oxysporum-initiated NO production. A transcriptional study of 78 NO-modulated genes indicated most genes were suppressed by single and combinational challenge with $F$. oxysporum or $T$. asperelloides. Only two $F$. oxysporum-induced genes were suppressed by $T$. asperelloides inoculation undertaken either $10 \mathrm{~min}$ prior to or after pathogen infection: a concanavlin A-like lectin protein kinase (At4g28350) and the receptorlike protein RLP30. Thus, $T$. asperelloides can actively suppress NO production elicited by $F$. oxysporum and impacts on the expression of some genes reported to be NO-responsive. Of particular interest was the reduced expression of receptor-like genes that may be required for $F$. oxysporumdependent necrotrophic disease development.

Trichoderma spp. are versatile opportunistic plant symbionts that can colonize the apoplast of plant roots. Trichoderma spp. limit the growth and activity of plant-pathogenic fungi by direct mycoparasitism. Some Trichoderma strains are capable of colonizing the entire root surface of different plant species, with a range of beneficial effects such as increases in growth, nutrient uptake, fertilizer efficiency utilization, seed germination, and establishing induced systemic resistance (Brotman et al. 2010, 2012; Shoresh et al. 2010). The wide range of effects caused by Trichoderma has led to the extensive use of Trichoderma-based agricultural formulations as a cost-effective ap-

Corresponding authors: Kapuganti J. Gupta; Telephone: +44 (0)1865 275024; E-mail: jagadis.kapuganti@plants.ox.ac.uk; and Yariv Brotman; E-mail: brotman@mpimp-golm.mpg.de; Telephone: +40 (0)331 5678273.

* The $e$-Xtra logo stands for "electronic extra" and indicates that three supplementary figures and one supplementary table are published online.

(C) 2014 The American Phytopathological Society proach to protect and improve yields of vegetable, ornamental, and fruit plantations (Viterbo and Horwitz 2010). The ability of Trichoderma to alleviate abiotic stresses is known, although specific knowledge of mechanisms through which multiple plant stress factors are affected is still lacking. Recently, Mastouri and associates (2012) demonstrated that T. harzianum T22treated tomato seedlings exhibited improved tolerance to water deficit by enhancing the antioxidant defense mechanisms through a higher activity of ascorbate and glutathione-recycling enzymes. Hence, it is likely that, at least in some Trichoderma strains, increased seedling vigor and amelioration of stress arise through increased protection from oxidative damage. Recently Brotman and associates (2013) demonstrated that salt stress tolerance induced by $T$. asperelloides (T203) is also dependent on activation of the plant antioxidant defense machinery, including an increased pool of reduced ascorbic acid.

Trichoderma penetration into the root tissue is usually limited to the first or second layers of cells and only to the intercellular spaces, leading to a strong defense response and programmed cell death (Yedidia et al. 1999). Using microarray analysis, Moran-Diez and associates (2012) recently showed global gene expression changes in aerial parts of Arabidopsis $24 \mathrm{~h}$ after root inoculation with T. harzianum T34. Most of the detected Arabidopsis defense-related genes were regulated by either jasmonic acid or salicylic acid and appeared to be downregulated by $T$. harzianum T34. T. harzianum-suppressed genes included those considered to be markers of systemic acquired resistance, e.g., FMO1 (flavin monooxygenase 1) and PR-1 (pathogenesis-related protein 1) (Pieterse et al. 1996). This suggests that Trichoderma spp., like the plant-beneficial fungus Piriformospora indica, suppress certain defense responses to allow its own colonization of Arabidopsis roots (Jacobs et al. 2011). It is relevant to note that certain "defense" responses are manipulated by some pathogens to promote disease (Pieterse et al. 2009); thus, counter-manipulation by Trichoderma spp. could suppress infection and disease development by certain pathogens.

Nitric oxide (NO) is important for regulation of numerous plant processes, such as growth and development, stomata function, adaptation to low or elevated temperatures, salt and water stress, and also in the induction of defense responses, (Besson Bard et al. 2008; Gupta 2011; Gupta et al. 2011a; Moreau et al. 2010). Along with the generation of ROS (reactive oxygen species) (Lamb and Dixon 1998), NO is rapidly generated in both incompatible and compatible plant-pathogen interactions (Delledonne et al. 1998; Mur et al. 2005; Zeidler et al. 2004). As with ROS, NO has also been linked to resistance against 
necrotrophic pathogens (Asai and Yoshioka 2009; Yoshioka et al. 2009), but with cell death being a feature of disease development by these pathogens-as is the case with Fusarium oxysporum (Bae et al. 2006) - NO generation could also act as a virulence effector (Turrion-Gomez and Benito 2011). More widely, NO is also generated during nonpathogenic plant microbial interactions, e.g., in nitrogen-fixing nodules forming during symbiotic interactions (Baudouin et al. 2006). For example, Medicago roots emit NO in contact with mycorrhizal exudates (Calcagno et al. 2012).

Different biosynthetic pathways for NO production are utilized in various compartments of the cell and under specific conditions (Gupta et al. 2011a). The most intensively studied enzyme involved in NO production is the cytosolic nitrate reductase (NR). The reaction of NR-dependent NO is NAD(P)H + $3 \mathrm{H}_{3} \mathrm{O}^{+}+2 \mathrm{NO}_{2}^{-} \rightarrow 72 \mathrm{NAD}^{+}+2 \mathrm{NO}+5 \mathrm{H}_{2} \mathrm{O}$. Various studies suggested that NR-mediated NO play a role in plant defense to various bacterial and fungal pathogens and elicitors (Gupta et al. 2011a; Shi and Li 2008; Srivastava et al. 2009, YamamotoKatou et al. 2006). In Arabidopsis, NR is encoded by two genes, NIA1 and NIA2. NR double nia mutants generate considerably less but still some NO, indicating the existence of other generation mechanisms. These alternative mechanisms include a root plasma membrane nitrite NO reductase (PM-NINOR) (Stohr and Ulrich 2002) or nitrite to NO reduction via complex IV cytochrome c oxidase (Gupta et al. 2011b, 2012). Plants are also known to produce NO by oxidative pathways such as the nitric oxide synthase (NOS)-like pathway or currently cryptic polyamine- and hydroxylamine-based routes (Corpas et al. 2009; Rümer et al. 2009; Tun et al. 2006). NOS-like activity has been shown during plant interaction with other microbes, especially with pathogens (Besson-Bard et al. 2008; Delledonne et al. 1998) and in plant development (Corpas et al. 2006).

Given the importance of NO in governing plant-pathogen interactions (Mur et al. 2005), we investigated its possible roles in the interaction of Trichoderma spp. with Arabidopsis roots and in combination with the important root pathogen $F$. oxysporum. We found a rapid and transient pattern of NO production in roots following contact with Trichoderma asperelloides that appeared to be dependent on NR. This pattern was markedly different from that elicited by the necrotrophic pathogen $F$. oxysporum. Coinoculation of $T$. asperelloides and $F$. oxysporum resulted in greatly reduced NO formation, which could be linked with a failure of the pathogens to initiate disease symptoms. This active repression of $T$. asperelloides-induced NO production was linked to key transcriptional changes on NO-responsive genes. This work provides key insights into the mechanisms of Trichoderma action to allow the optimal use of this beneficial fungus in sustainable agricultural practice.

\section{RESULTS AND DISCUSSION}

\section{T. asperelloides induces NO during early stages of contact.}

Inoculation of Arabidopsis roots with Fusarium oxysporum f. sp. lentis led to the formation of necrotic symptoms and extensive colonization within 3 days of infection (Supplementary Fig. 1A). Such necrotic symptoms are often linked to the initiation of plant defense and generation of ROS in early stages (Lamb and Dixon 1997; Zhu et al. 2013) and this was assessed by $2^{\prime}, 7^{\prime}$-dichlorofluorescin diacetate (DCF-2DA) fluorescence at 10, 30, 60, and 120 min following challenge. Roots infected with $F$. oxysporum led to a rapid and persistent generation of ROS (Fig. 1A). By 3 days after challenge, Arabidopsis roots infected with $T$. asperelloides (a nonpathogen) displayed relatively minor browning. Examining early responses in Arabidopsis roots to $T$. asperelloides suggested less ROS generation compared with that observed with $F$. oxysporum (Fig. 1B) that correlated with the lesser root necrosis seen at 3 days. Only a weak DCF signal was observed in uninfected roots (Fig. 1C).

NO and ROS are often simultaneously generated during plant-fungal interactions (Scheler et al. 2013). Thus, the patterns of NO production were determined in T. asperelloides and F. oxysporum interactions with Arabidopsis roots. Arabidopsis roots were infected with $T$. asperelloides, and NO pro-
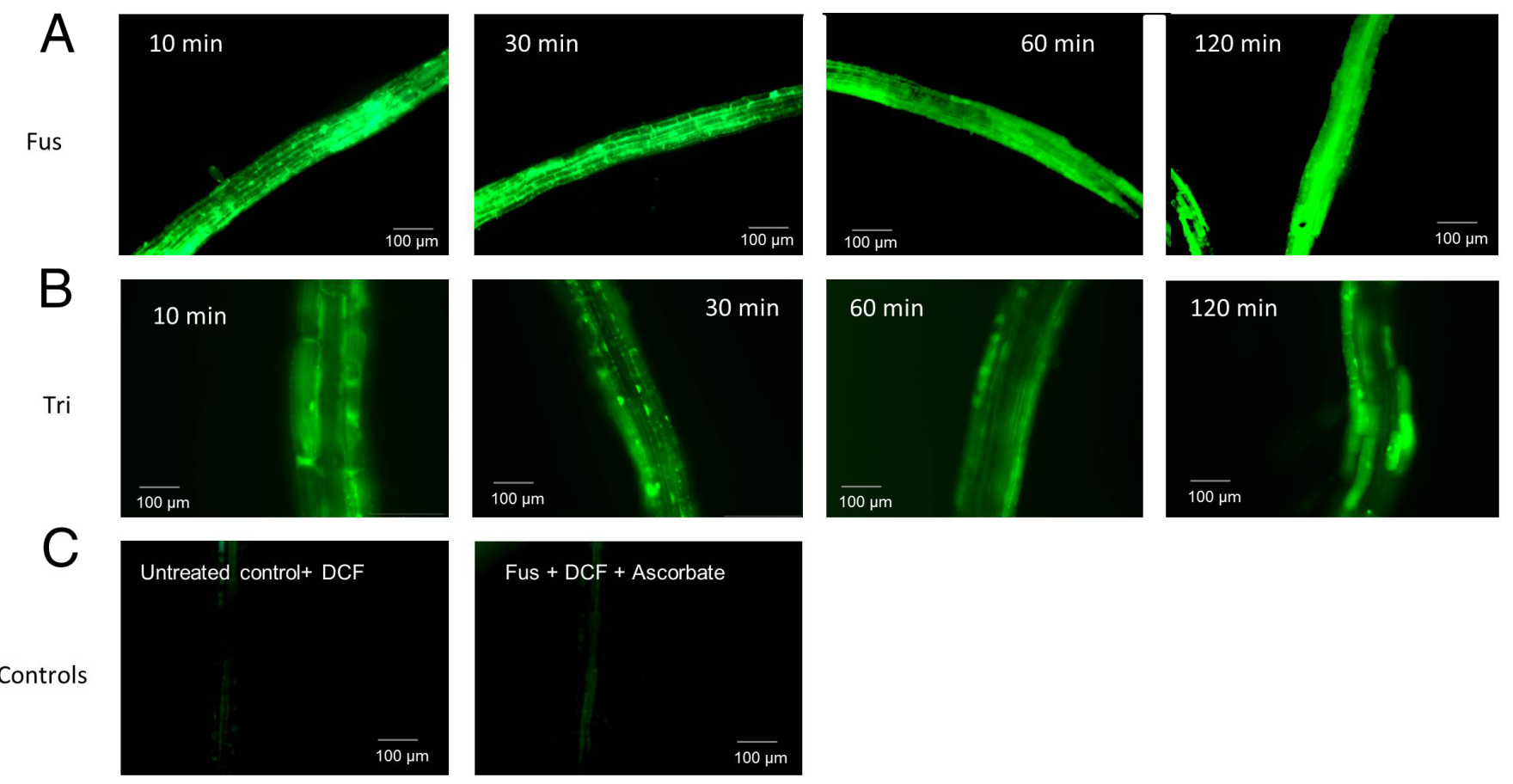

Fig. 1. Reactive oxygen species (ROS) levels visualized by $2^{\prime}, 7^{\prime}$-dichlorofluorescin (DCF) fluorescence from Arabidopsis roots. ROS generation following infection with either A, Fusarium oxysporum or B, Trichoderma asperelloides at different time points as indicated in the figure. C, Fluorescence from untreated control and ROS scavenger ascorbate incubated $F$. oxysporum-infected roots. Pictures show one independent representative of four biological replicates. Bars, $100 \mu \mathrm{m}$. 
duction pattern was monitored at various time intervals (10, 30,60 , and $120 \mathrm{~min}$ ) by fluorescence microscopy using NOspecific diaminofluorescein diactate (DAF-2DA). Intense fluorescence was observed after $10 \mathrm{~min}$ and was localized to discrete cells but was only weakly detected at $30 \mathrm{~min}$ and not at all thereafter (Fig. 2A). This pattern contrasted with that seen when challenging with $F$. oxysporum, when a steady NO fluorescence was observed over wide portions of the roots (Fig. 2B). Very low fluorescence was observed in the untreated control (Fig. 2C). Incubation of roots with $100 \mu \mathrm{M}$ cPTIO (2-(4-carboxyphenyl)-4, 4, 5, 5-tetramethylimidazoline-1-oxyl-3-oxide) abolished fluorescence induced by $T$. asperelloides, confirming that NO production was, indeed, being measured (Fig. 2C). Our inoculation techniques involved the addition of both fungal spores and mycelium, so the observed patterns of NO could, at least in part, be originating from the fungi. To check whether the observed NO was derived from $T$. asperelloides or $F$. oxysporum alone this was assessed using DAF2-DA staining (Supplementary Fig. 2). No NO production was observed from isolated $T$. asperelloides or $F$. oxysporum hyphae, suggesting that the NO observed in Figure 2A and B is derived from roots and not from the microorganism. Interestingly, weak DAF-2DA fluorescence was observed when both fungal species were combined, which could be the result of the production of antimicrobial compounds from T. asperelloides (Harman et al. 2004), initiating stress-associated NO production from $F$. oxysporum.

We sought to confirm the pattern of NO production induced by $T$. asperelloides and $F$. oxysporum by an alternative assay method. Thus, we measured nitrite content as an indirect measure of NO content using the Griess reagent assay. Arabidopsis roots exhibited a threefold increase in nitrite content in response to $T$. asperelloides the first $10 \mathrm{~min}$, before returning to background levels after 30, 60, and 120 min following inoculation (Fig. 3A), whereas F. oxysporum_inoculation led to a steady increase in nitrite over the 120-min experimental period (Fig. 3B). This pattern of NO production closely conformed to that seen when using the DAF-2DA assay.

\section{NR and not NOS is responsible for NO production in response to $T$. asperelloides.}

Previously, it was described that NR or NOS-like enzyme was responsible for NO production during plant-microbe inter-

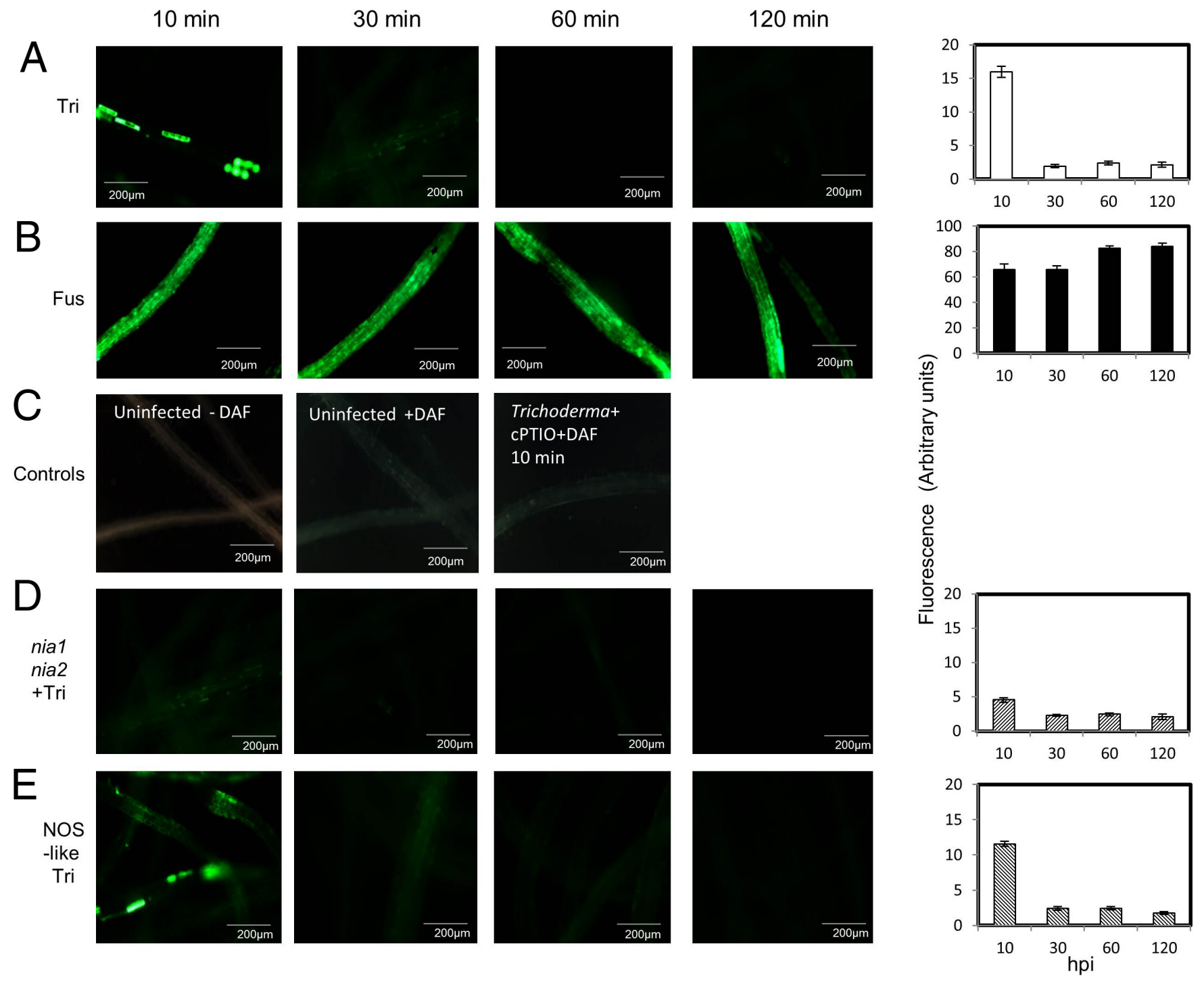

Fig. 2. Visualization of nitric oxide by diaminofluorescein (DAF) fluorescence at different time points. A, Nitric oxide (NO) production from wild-type (WT) (Col-0) roots treated with Trichoderma asperelloides. B, NO production from WT (Col-0) roots treated with Fusarium oxysporum. C, DAF fluorescence from untreated control and WT roots infected with T. asperelloides for $10 \mathrm{~min}$ in the presence of cPTIO. D, NO production from nitrate reductase double mutant (nial/nia2) treated with T. asperelloides. E, NO production from roots of WT grown on L-NAME. One independent representative of four biological replicates is shown. Right side of each panel of figures shows a quantitative view of fluorescence from pictures. Bars, $200 \mu \mathrm{m}$. 
actions (Gupta et al. 2011a). In order to test if NR activity was the source of NO production in roots in contact with $T$. asperelloides (Fig. 2A), we monitored NO production in the nial/nia2 double mutant at various time intervals matching those shown in Figure 2A. No detectable NO product occurred in nia double mutant roots, suggesting that NR might be responsible for the NO production (Fig. 2D). To ascertain if there was a contribution from a NOS-like enzyme to T. asperelloides-induced NO production, plants were treated with L-Arginine substrate analogues, which inhibit mammalian NOS activity. Application of $2.5 \mathrm{mM}$ L-NAME failed to significantly suppress NO formation in roots, clearly suggesting that NOS-like enzyme is not involved in NO production elicited by $T$. asperelloides (Fig. $2 \mathrm{E})$. Our results show that, at least in the case of root infections with T. asperelloides, NR is responsible for NO production.

\section{T. asperelloides represses $\boldsymbol{F}$. oxysporum-induced NO.}

Initial experimentation sought to establish that $T$. asperelloides could suppress $F$. oxysporum-associated disease development. Coinfection of Arabidopsis roots with T. asperelloides and $F$. oxysporum resulted in no significant disease symptom development after 3 days. Given these observations, the effects of $T$. asperelloides on $F$. oxysporum-elicited patterns of $\mathrm{NO}$ production were investigated.

Initially, roots were inoculated with $T$. asperelloides and $F$. oxysporum simultaneously, and NO generation assessed after 10 and 120 min (Fig. 4C and D, respectively). Although F. oxysporum induced NO production for at least $120 \mathrm{~min}$ (Fig. 2B), this was hardly detectable if the roots were coinoculated with $T$. asperelloides. However, at $10 \mathrm{~min}$, NO generation was detected, although this appeared to match the cell-specific pattern seen with T. asperelloides rather than the more intense diffusion pattern elicited by F. oxysporum (Fig. 4A compared with B). Griess assays were used to confirm this pattern of NO production. Incubation of roots with $T$. asperelloides plus $F$. oxysporum led to a threefold increase in nitrite levels, which returned to background levels after 30, 60, and 120 min following inoculation (Fig. 3C), consistent with nitrite levels observed in the presence of $T$. asperelloides alone (Fig. 3A). To further investigate whether this residual NO production was an elicitory effect of $T$. asperelloides or F. oxysporum, a series of staggered inoculations were undertaken. Thus, Arabidopsis roots were inoculated with T. asperelloides, and then, after $10 \mathrm{~min}$, with $F$. oxysporum, and $\mathrm{NO}$ production was assessed after a further $120 \mathrm{~min}$. At this time point, any $T$. asperelloides-elicitory events would have expected to have dissipated (Fig. 2A) but would be maintained if $F$. oxysporum were influencing the pattern of NO generation. At the 30-min time point (10 min T. asperelloides $+20 \mathrm{~min} F$. oxysporum), some NO production was detected (Fig. 4E) but it was greatly reduced compared with $F$. oxysporum alone (Fig. 2B). This greatly suppressed pattern of NO production was maintained until at least 120 min following a second inoculation with
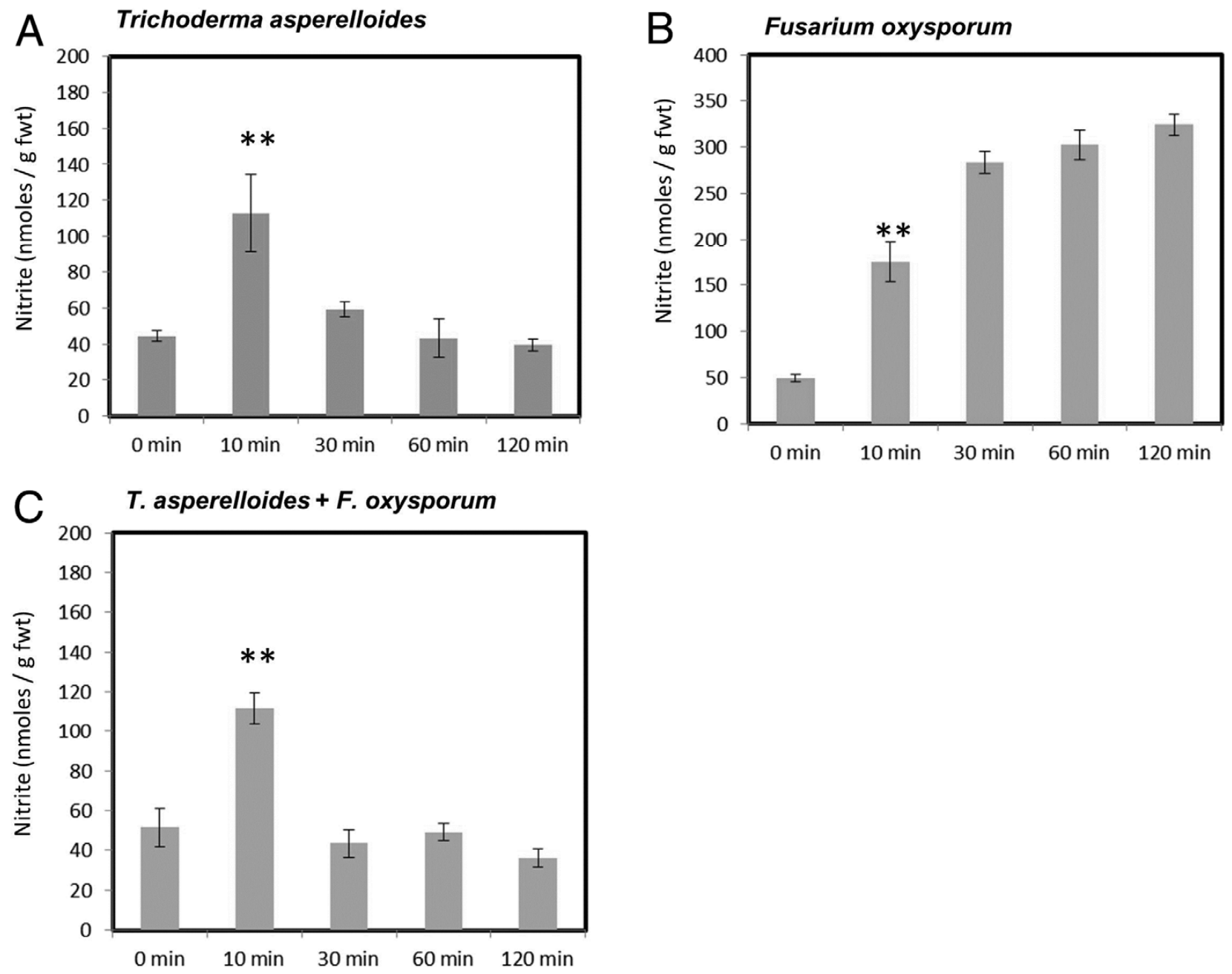

Fig. 3. Griess assays reporting nitric oxide production in the interactions of Trichoderma asperelloides and Fusarium oxysporum with Arabidopsis roots Nitrite content in roots infected with A, T. asperelloides, B, F. oxysporum, and C, T. asperelloides plus F. oxysporum at 0 , 10 , $30,60,120$ min. Results showing a significant $(P<0.05)$ increase in $10 \mathrm{~min}$ is indicated by double asterisks $(* *)$. 
F. oxysporum (Fig. 4F as compared with B). These results suggest that $T$. asperelloides can suppress NO production and block the elicitation of NO by F. oxysporum.

In order to test if an early $F$. oxysporum NO-elicitory mechanism was blocked, we performed a reciprocal study in which we elicited NO formation by $F$. oxysporum infection followed, after $20 \mathrm{~min}$, by $T$. asperelloides inoculation. In this case, NO production induced by $F$. oxysporum was also suppressed (Fig. $4 \mathrm{G}$ and $\mathrm{H}$ ), indicating that $T$. asperelloides was acting by blocking the generation of NO following recognition of $F$. oxysporum by the host. Given the rapidity of these responses, it seems likely that they, at least in part, reflect responses to preformed elicitors on the fungal spores, hyphal, or secreted elicitors (Bae et al. 2006; Hanson and Howell 2004; Woo et al. 2006), which would not be distinguished by our inoculation technique.

To assess whether T. asperelloides-mediated suppression of NO production was effective against other pathogens, we tested NO levels in response to two different pathogens, i.e., Verticillium dahlia and virulent Pseudomonas syringae pv. tomato DC3000, that can attack roots. In both cases, NO levels rapidly increased in 10, 30, 60, and 120 minutes (Supplementary Fig. $3 \mathrm{~A}$ and B). Simultaneous inoculation of $T$. asperelloides with either Verticillium dahlia or P. syringae pv. tomato DC3000 led to a suppression of $\mathrm{NO}$, suggesting that $T$. asperelloides has the ability to suppress NO production induced by a wide range of pathogens.

\section{T. asperelloides can actively suppress NO production elicited} by $F$. oxysporum to perturb NO-regulated genes linked to infection with $F$. oxysporum.

To characterize the T. asperelloides suppressive mechanism on NO effects, we analyzed the expression of 78 genes that had been shown to be induced by either NO gas or NO donors (Palmieri et al. 2008, Parani et al. 2004; Polverari et al. 2003) (Supplementary Table 1). These genes included many genes involved in metabolite detoxification (such as glutathione $S$-transferases) and the production of secondary metabolites or have established functions in defense. To bias the analyses towards $T$. asperelloides suppressive events, our gene-expression screen were based on our experimental staggered $T$. asperelloides and F. oxysporum inoculation approach. The expression level of these NO-modulated genes was followed using quantitative reverse transcription-polymerase chain reaction (qRT-PCR) in Arabidopsis roots elicited with either i) T. asperelloides for 10 min, ii) F. oxysporum for $10 \mathrm{~min}$, iii) T. asperelloides for $10 \mathrm{~min}$,
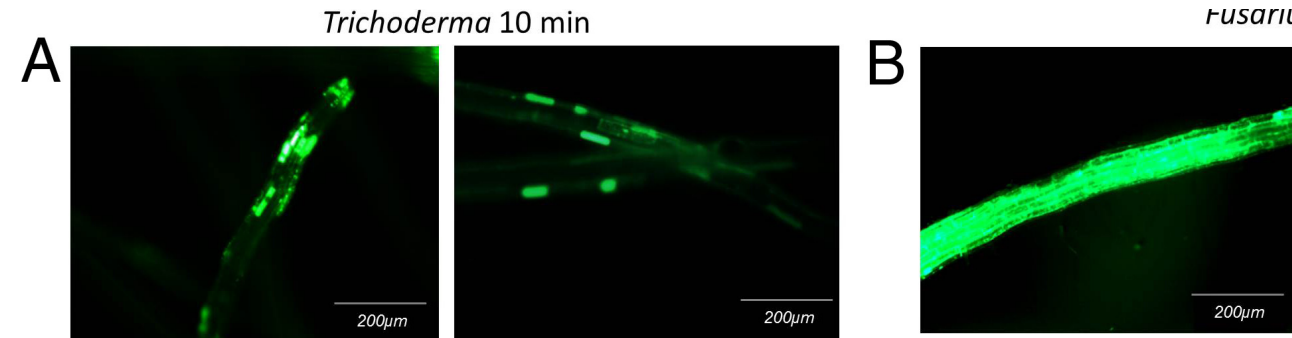

rusarıum iU mın
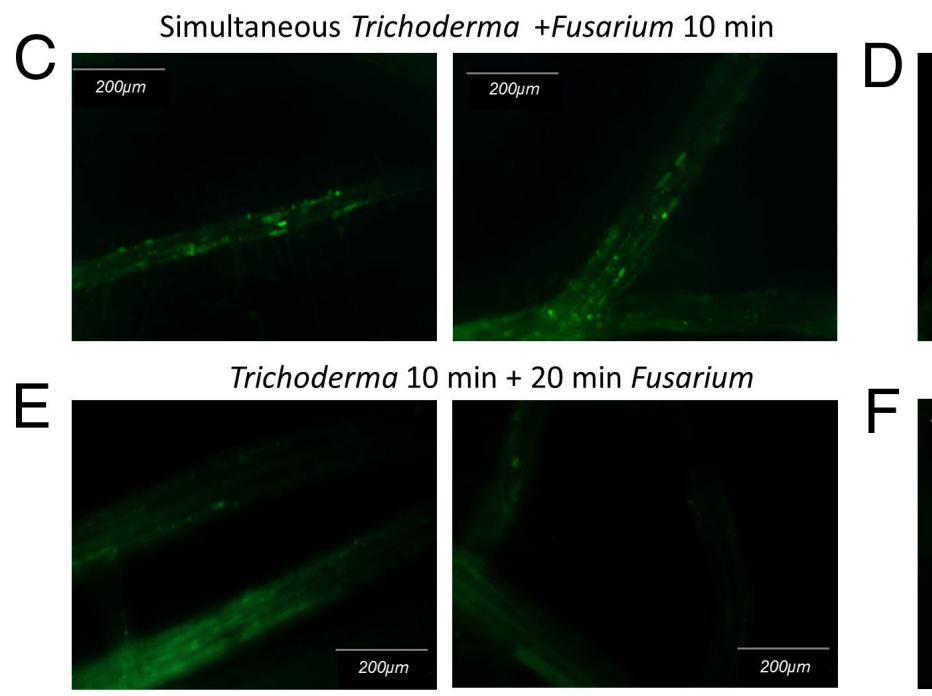

Simultaneous Trichoderma +Fusarium $120 \mathrm{~min}$
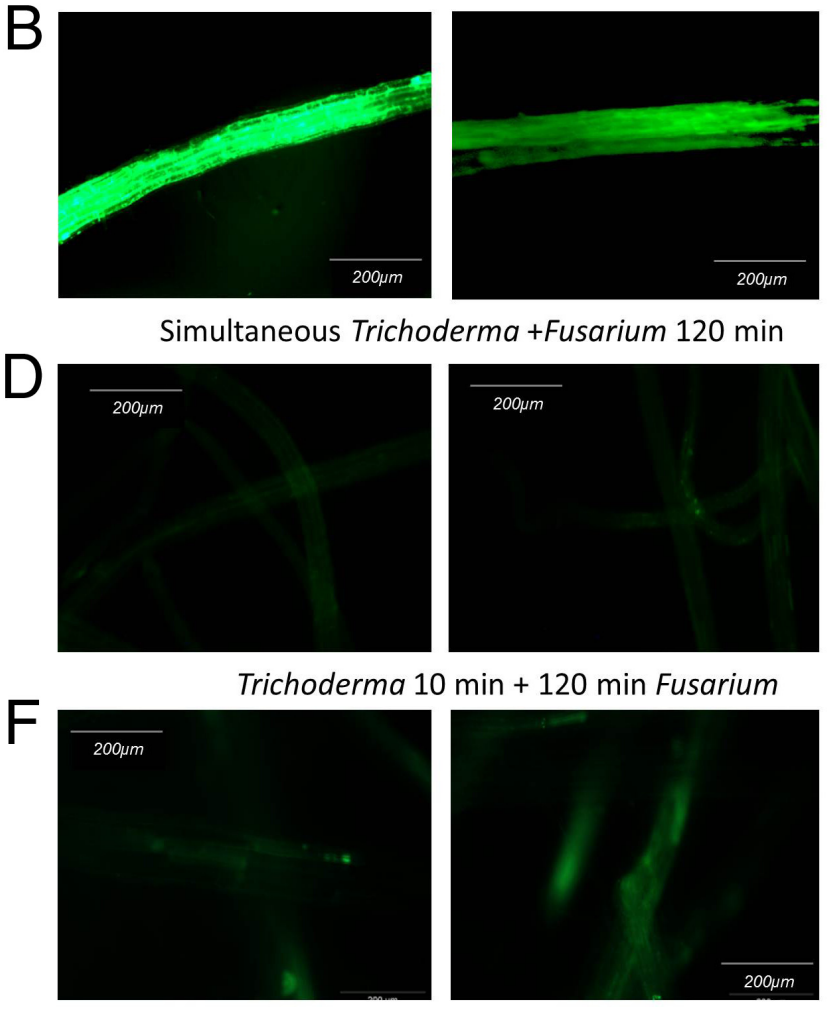

Fusarium $10 \mathrm{~min}+$ Trichoderma $20 \mathrm{~min}$
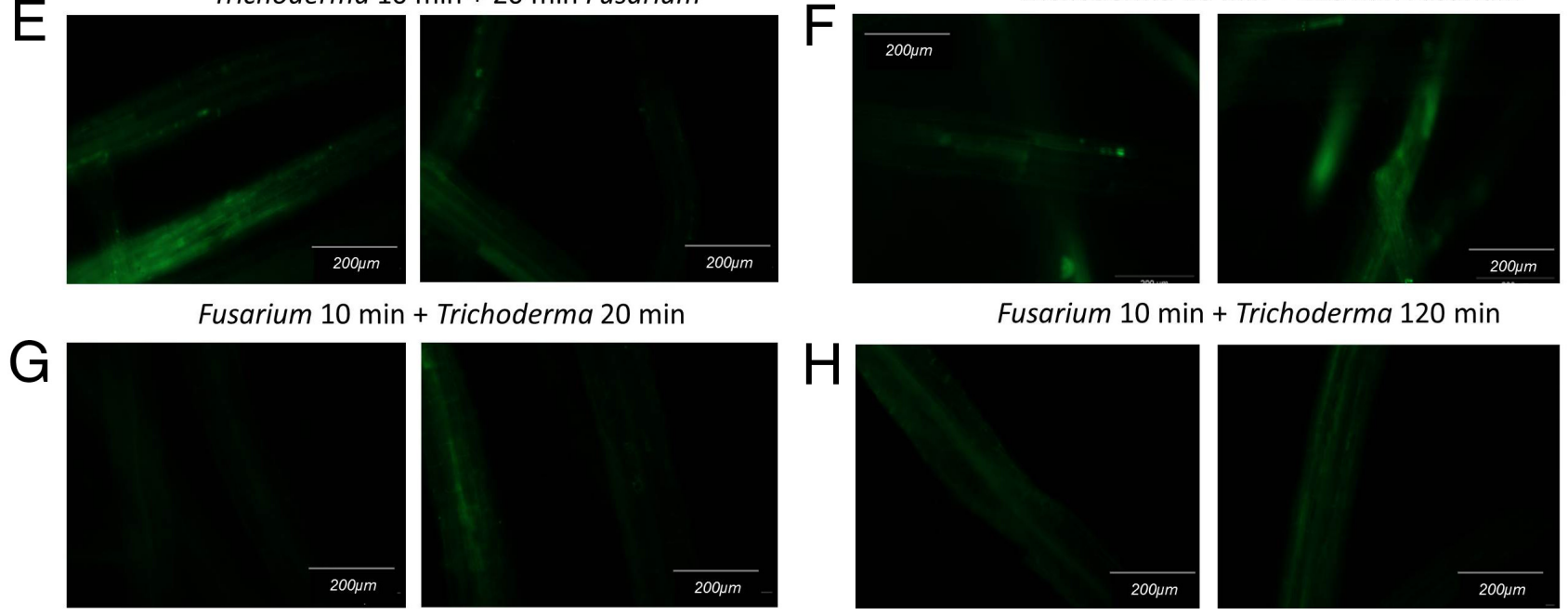

Fusarium $10 \mathrm{~min}+$ Trichoderma $120 \mathrm{~min}$

Fig. 4. Nitric oxide (NO) production from Arabidopsis following coinoculation with Trichoderma asperelloides and Fusarium oxysporum. NO production in wild-type (WT) roots in 10 min treated with $\mathbf{A}, T$. asperelloides or $\mathbf{B}, F$. oxysporum. $\mathbf{C}$, NO production from WT roots simultaneously treated with $F$. oxysporum and $T$. asperelloides at $10 \mathrm{~min}$ and $\mathbf{D}, 120 \mathrm{~min}$. E, NO production from WT roots treated with T. asperelloides followed at $10 \mathrm{~min}$ by $F$. oxysporum assessed at $20 \mathrm{~min}$ and $\mathbf{F}, 120 \mathrm{~min}$. G, NO production from WT roots treated with F. oxysporum followed at $10 \mathrm{~min}$ by $T$. asperelloides with NO production assessed at $20 \mathrm{~min}$ and $\mathbf{H}$, at $120 \mathrm{~min}$.One independent representative of four biological replicates in all treatments is shown. Bars, $200 \mu \mathrm{m}$. 
followed by $F$. oxysporum for $60 \mathrm{~min}$, and iv) $F$. oxysporum for $10 \mathrm{~min}$, followed by $T$. asperelloides for $60 \mathrm{~min}$. Hierarchical clustering (Fig. 5A) indicated considerable variation in the expression of these NO-regulated genes that did not always match the observed patterns of NO generation (Fig. 2). This indicated that NO was not the sole factor governing their relative transcriptional patterns. Most of the assessed genes exhibited a reduction in gene expression following interaction with either microbial species. Those genes displaying the most differential responses to the various treatments are displayed in Figure 5.

Examining distinctive transcriptional responses to $F$. oxysporum, it was noted that alternative oxidase 1A (At3g23370) and $\mathrm{Fe}$ (II)-dependent oxygenase superfamily protein (At5g05600) were rapidly (10 $\mathrm{min})$ but transiently (i.e. not expressed at $60 \mathrm{~min}$ ) expressed (Fig. 5). A jasmonate-synthesizing lipoxygenase 3 (LOX3; At1g1742) was induced only by F. oxysporum at 60 min (Fig. 5). A toll interleukin 1 receptornucleotide binding site-leucine-rich repeat disease-resistance gene (At1g63750), 2-oxoglutarate (2OG; At2g38240), a stress responsive phloem protein 2 A5 (At1g65390), and a FADbinding Berberine family protein (At4g20830) were transiently (i.e., expressed only at $10 \mathrm{~min}$ following interaction) expressed with T. asperelloides. At $60 \mathrm{~min}, T$. asperelloides-induced transcripts were a different $20 \mathrm{G}$ gene (At3g19010), RGP1
(At3g02230), a tau-class glutathione $S$-transferase (At2g29940), and a stress-regulating chaperone DnaJ-domain superfamily protein (Chen et al. 2010). Such patterns were consistent with complex responses to individual inoculation with each fungal species that have been explored elsewhere (Moran-Diez et al. 2012; Zhu et al. 2013).

Most relevant to this current study were those genes whose expression was modulated by $F$. oxysporum but whose expression was either countered by either prior or postinoculation with T. asperelloides (Fig. 5). LOX3, (At1g17420), previously induced by $F$. oxysporum, was now suppressed with $T$. asperelloides infection. Expression of the glycine decarboxylase Pprotein 1 (GLDP1; At4g33010) was suppressed by both F. oxysporum and $T$. asperelloides alone but dual inoculation with both fungal species elevated expression. In the case of alternative oxidase $(A O X 1 a)$, prior inoculation with $T$. asperelloides led to increased expression after $60 \mathrm{~min}$ following infection with $F$. oxysporum.

Interestingly, all of the genes that were induced at $60 \mathrm{~min}$ after inoculation with T. asperelloides-At4g36040, At3g19010, At2g29440, At3g02230 - were apparently suppressed by prior or postinoculation with $F$. oxysporum. With Atg21870 (a member of the Hsp20 family of proteins) and At5g53450 (an ACC oxidase), both genes were induced by dual inoculations, whereas
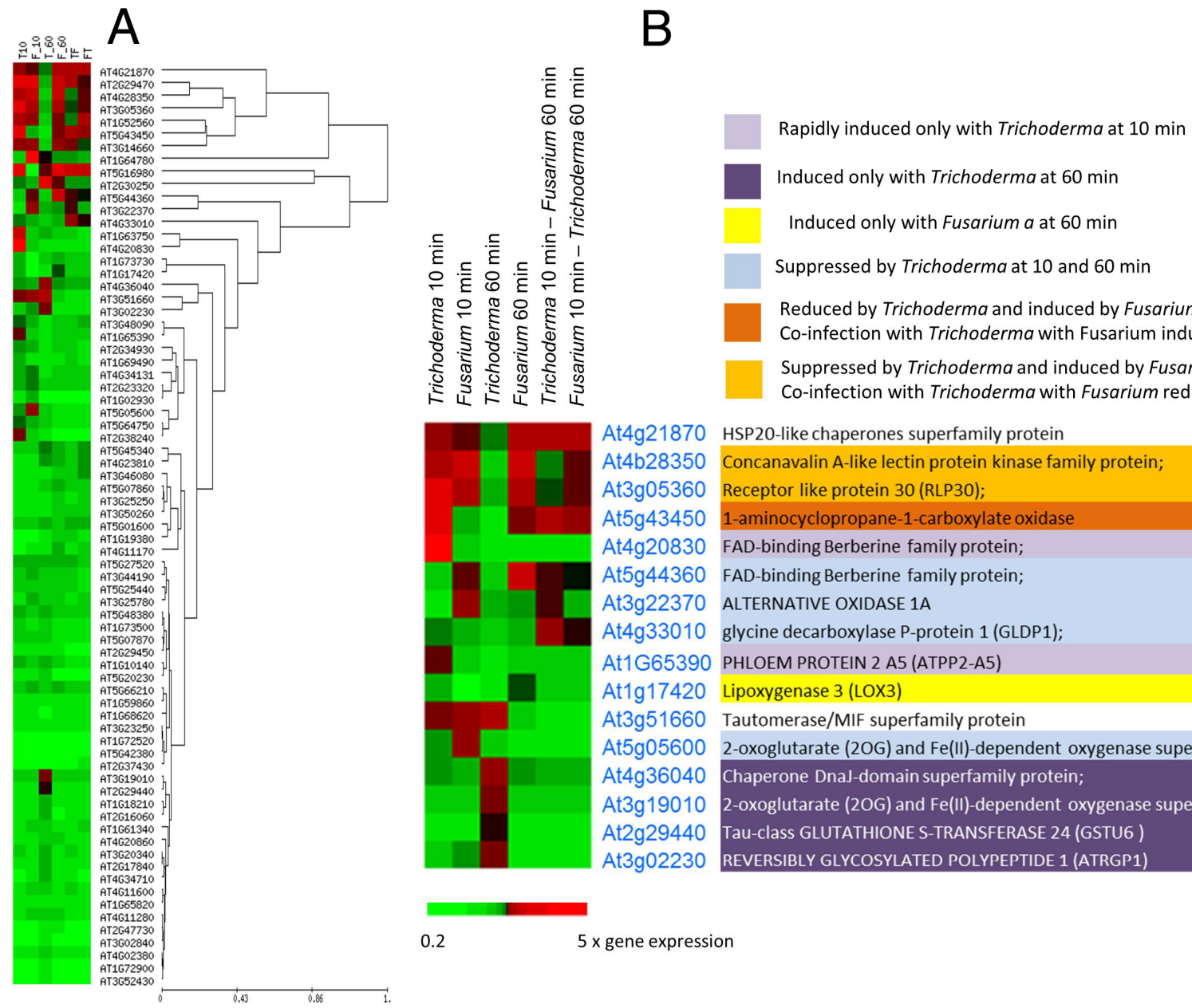

\section{Suppressed by Trichoderma at 10 and $60 \mathrm{~min}$}

Reduced by Trichoderma and induced by Fusarium at $60 \mathrm{~min}$. Co-infection with Trichoderma with Fusarium induced expression at $60 \mathrm{~min}$

Suppressed by Trichoderma and induced by Fusarium at $60 \mathrm{~min}$.

Co-infection with Trichoderma with Fusarium reduces expression at $60 \mathrm{~min}$

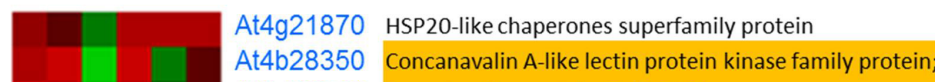

At4b28350 Concanavalin A-like lectin protein
At3g05360 Receptor like protein 30 (RLP30);

At5g43450 1-aminocyclopropane-1-carboxylate oxidase

At4g20830 FAD-binding Berberine family protein;

At5g44360 FAD-binding Berberine family protein:

At3g22370 ALTERNATIVE OXIDASE $1 \mathrm{~A}$

At4g33010 glycine decarboxylase P-protein 1 (GLDP1);

At1G65390 PHLOEM PROTEIN 2 A5 (ATPP2-A5)

At1g17420 Lipoxygenase 3 (LOX3)

At3g51660 Tautomerase/MIF superfamily protein

At5g05600 2-oxoglutarate (2OG) and Fe(II)-dependent oxygenase superfamily protein;

At4g36040 Chaperone DnaJ-domain superfamily protein;

At3g19010 2-oxoglutarate (2OG) and Fe(II)-dependent oxygenase superfamily protein;

At2g29440 Tau-class GLUTATHIONE S-TRANSFERASE 24 (GSTU6)

At3g02230 REVERSIBLY GLYCOSYLATED POLYPEPTIDE 1 (ATRGP1)

Fig. 5. Transcriptional regulation of various genes in response to inoculation by Trichoderma asperelloides and Fusarium oxysporum. Arabidopsis roots were incubated with one or both $T$. asperelloides or F. oxysporum and were sampled at different times: i) inoculating with T. asperelloides and sampling after 10 min; ii) inoculating with F. oxysporum and sampling after $10 \mathrm{~min}$; iii) inoculating with $T$. asperelloides and sampling after $60 \mathrm{~min}$; iv) inoculating with $F$. oxysporum and sampling after $60 \mathrm{~min} ; \mathrm{v}$ ) inoculation first with T. asperelloides, followed at $10 \mathrm{~min}$ by inoculation with $F$. oxysporum and sampled at 60 min. A, The expression of NO-regulated genes were derived following quantitative reverse transcriptas-polymerase chain reaction and were used in a hierarchical cluster analysis. Gene expression values $\left(\log _{2}\right)$ of the genes are indicated using a heat map. B, Clusters of genes color coded to highlight the patterns of gene expression across the treatments. 
these were suppressed when inoculating with $T$. asperelloides alone after $60 \mathrm{~min}$. Taken together, these patterns could suggest that dual inoculations reduced stress-linked events with possibly increased ethylene but less production of jasmonates.

Of special interest was the induction of At $4 \mathrm{~g} 28350$ and At3g05360, both of which are defense-associated receptor kinases (Navarro et al. 2004; Wang et al. 2008), by F. oxysporum after $60 \mathrm{~min}$. The expression of both was reduced by prior or postinoculation with $T$. asperelloides. This was consistent with the elicitation by $F$. oxysporum of certain defense-associated events being blocked or suppressed or both by $T$. asperelloides.

Our observations provide novel insights into the interactions of Trichoderma spp. with its plant host and competition with other interacting microbes that should stimulate further work by ourselves and other groups. An important finding was that T. asperelloides has the ability to switch off NR-linked NO production elicited by $F$. oxysporum. The timings of these events could suggest that mechanisms that initiate or maintain $F$. oxysporum-elicited NR production are being modified by $T$. asperelloides inoculation. Undoubtedly, these will include altered signaling events focusing on NR activation, which we will focus upon in subsequent studies. Our strategy was to examine the immediate $(10 \mathrm{~min})$ and longer term $(60 \mathrm{~min})$ effects on NO-mediated gene expression in an attempt to assess the NO component in the T. asperelloides-disease suppression. The definition of NO-responsive genes that are suppressed by T. asperelloides represents an initial stage in understanding a previously unsuspected facet of its action. These genes could also act as biomarkers in the development of new and more effective pathogen-suppressive $T$. asperelloides strains or even plant germplasm that is either more tolerant of $F$. oxysporum infections or represent better hosts for T. asperelloides.

\section{MATERIALS AND METHODS}

\section{Plant cultivation and inoculation.}

Arabidopsis thaliana (Col-0) plants were grown, horizontally, for 12 days under long day conditions, on Murashige Skoog $1.5 \%$ agar plates. $T$. asperelloides T203 and F. oxysporum f. sp. lentis were grown on potato dextrose agar plates. Plant inoculation involved taking a loop-full of inoculum, consisting of both mycelium and spores, which was added to a 2-ml microcentrifuge tube in which the plant intact root system was placed. The experiment included the following treatments: i) untreated plants (control); ii) plants challenged with T. asperelloides for 10, 20, 30, 60, or $120 \mathrm{~min}$; iii) F. oxysporum-treated plants for $10,20,30,30,60$, or $120 \mathrm{~min}$; iv) plants simultaneously challenged with $T$. asperelloides and $F$. oxysporum; v) plants challenged with $F$. oxysporum for $10 \mathrm{~min}$, and then, with $T$. asperelloides; vi) plants challenged with $T$. asperelloides for $10 \mathrm{~min}$, and then, with $F$. oxysporum. For gene expression analysis by qPCR, roots were collected at the different time points and shock-frozen with liquid nitrogen.

\section{Expression profiling by qRT-PCR.}

Expression analyses were performed using an expression profiling platform covering 78 genes that were selected followed a literature survey for NO-responsive genes in Arabidopsis (Besson-Bard et al. 2009; Palmieri et al. 2008). Primer sequences were designed using the QuantPrime online tool. Roots were collected and pooled from 20 plants in each treatment. Total RNA was purified using the RNeasy mini kit (Qiagen, Hilden, Germany) and DNaseI digestion was performed with the Turbo DNA-free Kit (Ambion, Austin, TX, U.S.A.). Four micrograms of total RNA were used as template for firststrand cDNA synthesis with the RevertAid cDNA synthesis kit (Fermentas, Vilnius, Lithuania) cDNA (20 ng) was used for
qPCR with Power SYBR Green reagent performed on an ABI PRISM 7900HT sequence detection system (Applied Biosystems, Foster City, CA, U.S.A.). Data were analyzed with the 7900 V2.0.3 evaluation software (Applied Biosystems). The fold change in the target genes was normalized to ACTIN2 and GADPH reference genes. Fold expression relative to control plants was determined using the cycle threshold method $(\Delta \Delta \mathrm{CT})$ as described in Libault and associates (2007). Three biological experiments (with two independent replicates for each experiment) were performed for each treatment. Comparisons of the gene expression between the different treatments to control used the Tukey multiple pairwise comparison test using Minitab v.14 (Minitab Ltd, Coventry, U.K.). Differences with $P<0.05$ were considered significant.

\section{Measurement of ROS and NO.}

Roots were incubated in $1 \mathrm{ml}$ of detection buffer DB $(2.5$ mM HEPES, pH 7.4) containing $10 \mu \mathrm{M}$ DAF-2DA (ENZO Biosciences, Farmingdale, NY, U.S.A.).The formation of DAF-2T following NO reaction with DAF-2DA was visualized at different time points, using a Leica fluorescent microscope upon excitation at $488 \mathrm{~nm}$ with an Argon 2 laser. Fluorescence emission was recorded using a 505- to 530-nm band-pass filter coupled with a 515-nm long-pass filter. Images were analyzed using the Meta software. To measure DCF fluorescence, infected roots were taken, $10 \mu \mathrm{M}$ DCF-2DA fluorescent dye (Invitrogen, Carlsbad, CA, U.S.A.) was added to the roots in the detection buffer, and the images were checked at various times as described for NO. To demonstrate NO was being measured, $200 \mu \mathrm{M}$ of the NO scavenger cPTIO was used as control. To determine if NOS-like enzyme was generated NO, $2.5 \mathrm{mM}$ arginine analogs NG-nitro-L-arginine methyl ester (LNAME) or L-NG-monomethyl-arginine monoacetate (LNMMA) were added to the medium of plant culture medium and, then, were transferred for 3 to 4 days into the inhibitor and fluorescence was quantified as described above. To confirm ROS generation, $10 \mu \mathrm{M}$ ascorbate was added to roots prior to the infection.

\section{Nitrite levels from Arabidopsis roots treated with T. asperelloides (Griess assay).}

Nitrite levels were measured according to Planchet and associates (2005). Aliquots of the above roots were sampled and quickly mixed with a reaction mixture containing $600 \mu \mathrm{l}$ of sulphanilamide (1\%), $600 \mu \mathrm{l}$ of $N$-(1)-(naphthyl) ethylenediaminedihydrochloride $(0.02 \%)$, and $300 \mu$ lof zinc acetate $(0.5 \mathrm{M})$. After $25 \mathrm{~min}$ of incubation at $24^{\circ} \mathrm{C}$, the mixture was cleared by centrifugation $(16,000 \times g, 5 \mathrm{~min})$, and the nitrite content from the supernatant was determined with a spectrophotometer.

\section{ACKNOWLEDGMENTS}

We specially thank L. Willmitzer for valuable suggestions and great support during this work. We thank A. Viterbo for comments on the text. This research was partially supported by the Deutsche ForschungsgemeinschaftIsrael Tri Lateral Cooperation Project between Germany, Israel, and the Palestinian Authority grant number 0306458 (Y. Brotman, L. A. J. Mur); Max Planck Society (K. J. Gupta; Y. Brotman) and Marie Curie Intra European Fellowship for Carrier Development within the 7th European Community Framework Programme (K. J. Gupta) and the BBSRC-DEFRA-HGCA SCORE LINK grant (L. A. J. Mur). We thank S. Datta for helpful discussions and help in microscopy. We thank the reviewers for their valuable comments which helped to improve the quality of this manuscript.

\section{LITERATURE CITED}

Asai, S., and Yoshioka, H. 2009. Nitric oxide as a partner of reactive oxygen species participates in disease resistance to necrotrophic pathogen 
Botrytis cinerea in Nicotiana benthamiana. Mol. Plant-Microbe Interact. 22:619-629.

Bae, H., Kim, M. S., Sicher, R. C., Bae, H. J., and Bailey, B. A. 2006. Necrosis- and ethylene-inducing peptide from $F$. oxysporum arium oxysporum induces a complex cascade of transcripts associated with signal transduction and cell death in Arabidopsis. Plant Physiol. 141:1056-1067.

Baudouin, E., Pieuchot, L., Engler, G., Pauly, N., and Puppo, A. 2006. Nitric oxide is formed in Medicago truncatula-Sinorhizobium meliloti functional nodules. Mol. Plant-Microbe Interact. 19:970-975.

Besson-Bard, A., Pugin, A., and Wendehenne, D. 2008. New insights into NO oxide signaling in plants. Annu. Rev. Plant Biol. 59:21-39.

Brotman, Y., Kapuganti, J. G., and Viterbo, A. 2010. Trichoderma. Curr. Biol. 20:R390-391.

Brotman, Y., Lisec, J., Meret, M., Chet, I., Willmitzer, L., and Viterbo, A. 2012. Transcript and metabolite analysis of the Trichoderma-induced systemic resistance response to Pseudomonas syringae in Arabidopsis thaliana. Microbiology 158:139-146.

Brotman, Y., Landau, U., Cuadros-Inostroza, A., Takayuki, T., Fernie, A. R., Chet, I., Viterbo, A., and Willmitzer, L. 2013. Trichoderma-plant root colonization: escaping early plant defense responses and activation of the antioxidant machinery for saline stress tolerance. PLoS Pathog. 9:e1003221. Published online.

Calcagno, C., Novero, M., Genre, A., Bonfante, P., and Lanfranco, L. 2012. The exudate from an arbuscular mycorrhizal fungus induces nitric oxide accumulation in Medicago truncatula roots. Mycorrhiza 22:259-269.

Chen, K. M., Holmstrom, M., Raksajit, W., Suorsa, M., Piippo, M., and Aro, E. M. 2010. Small chloroplast-targeted DnaJ proteins are involved in optimization of photosynthetic reactions in Arabidopsis thaliana. BMC Plant Biol. 10:43.

Corpas, F. J., Palma, J. M., del Rio, L. A., and Barroso, J. B. 2009. Evidence supporting the existence of L-arginine-dependent nitric oxide synthase activity in plants. New Phytol. 184:9-14.

Corpas, F. J., Barroso, J. B., Carreras, A., Valderrama, R., Palma, J. M., Leon, A. M., Sandalio, L. M., and del Rio, L. A. 2006. Constitutive arginine-dependent nitric oxide synthase activity in different organs of pea seedlings during plant development. Planta 224:246-254.

Delledonne, M., Xia, Y. J., Dixon, R. A., and Lamb, C. 1998. Nitric oxide functions as a signal in plant disease resistance. Nature 394:585-588.

Gupta, K. J. 2011. Protein S-nitrosylation in plants: Photorespiratory metabolism and NO signaling. Sci Signal 4

Gupta, K. J., Fernie, A. R., Kaiser, W. M., and van Dongen, J. T. 2011a. On the origins of nitric oxide. Trends Plant Sci. 16:160-168.

Gupta, K. J., Igamberdiev, A. U., Manjunatha, G., Segu, S., Moran, J. F. Neelawarne, B., Bauwe, H., and Kaiser, W. M. 2011b. The emerging roles of nitric oxide (NO) in plant mitochondria. Plant Sci. 181:520-526

Gupta, K. J., Shah, J. K., Brotman, Y., Jahnke, K., Willmitzer, L., Kaiser, W. M., Bauwe, H., and Igamberdiev, A. U. 2012. Inhibition of aconitase by nitric oxide leads to induction of the alternative oxidase and to a shift of metabolism towards biosynthesis of amino acids. J. Exp. Bot. 63:1773-1784.

Hanson, L. E., and Howel, C. R. 2004. Elicitors of plant defense responses from biocontrol strains of Trichoderma viren. Phytopathology 94:171176.

Harman, G..E., Howell, C..R., Viterbo, A., Chet, I., and Lorito, M. 2004. Trichoderma species-Opportunistic, avirulent plant symbionts. Nat. Rev. Microbiol. 2:43-56.

Jacobs, S., Zechmann, B., Molitor, A., Trujillo, M., Petutschnig, E., Lipka, V., Kogel, K. H., and Schafer, P. 2011. Broad-spectrum suppression of innate immunity is required for colonization of Arabidopsis roots by the fungus Piriformospora indica. Plant Physiol. 157:531-531.

Lamb, C., and Dixon, R. A. 1997. The oxidative burst in plant disease resistance. Ann. Rev. of Plant Physiol. Plant Mol. Biol. 48:251-275.

Libault, M., Wan, J., Czechowski, T., Udvardi, M., and Stacey, G. 2007. Identification of 118 Arabidopsis transcription factor and 30 ubiquitinligase genes responding to chitin, a plant-defense elicitor. Mol. PlantMicrobe Interact. 20:900-911.

Mastouri, F., Björkman, T., and Harman, G. E. 2012. Trichoderma harzianum enhances antioxidant defense of tomato seedlings and resistance to water deficit. Mol. Plant-Microbe Interact. 25:1264-1271.

Moran-Diez, E., Rubio, B., Dominguez, S., Hermosa, R., Monte, E., and Nicolas, C. 2012. Transcriptomic response of Arabidopsis thaliana after $24 \mathrm{~h}$ incubation with the biocontrol fungus Trichoderma harzianum. J. Plant Physiol. 169:614-620.

Moreau, M., Lindermayr, C., Durner, J., and Klessig, D.F. 2010. NO synthesis and signaling in plants-Where do we stand? Physiol. Plantarum 138:372-383.

Mur, L. A. J., Santosa, I. E., Laarhoven, L. J. J., Holton, N. J., Harren, F. J. M., and Smith, A. R. 2005. Laser photoacoustic detection allows in planta detection of nitric oxide in tobacco following challenge with avirulent and virulent Pseudomonas syringae pathovars. Plant Physiol. 138:1247-1258.

Navarro, L., Zipfel, C., Rowland, O., Keller, I., Robatzek, S., Boller, T., and Jones, J. D. G. 2004. The transcriptional innate immune response to flg22, interplay and overlap with Avr gene-dependent defense responses and bacterial pathogenesis. Plant Physiol. 135:1113-1128.

Palmieri, M. C., Sell, S., Huang, X., Scherf, M., Werner, T., Durner, J., and Lindermayr, C. 2008. Nitric oxide-responsive genes and promoters in Arabidopsis thaliana: A bioinformatics approach. J. Exp. Bot. 59:177186.

Parani, M., Rudrabhatla, S., Myers, R., Weirich, H., Smith, B., Leaman, D. W., and Goldman, S. L. 2004. Microarray analysis of nitric oxide responsive transcripts in Arabidopsis. Plant Biotechnol. J. 2:359-366.

Pieterse, C. M. J., vanWees, S. C. M., Hoffland, E., van Pelt, J. A., and van Loon, L. C. 1996. Systemic resistance in Arabidopsis induced by biocontrol bacteria is independent of salicylic acid accumulation and pathogenesis-related gene expression. Plant Cell 8:1225-1237.

Pieterse, C. M., Leon-Reyes, A., Van der Ent, S., and Van Wees, S. C. 2009. Networking by small-molecule hormones in plant immunity. Nat. Chem. Biol. 5: 308-316.

Planchet, E., Gupta, K. J., Sonoda, M., and Kaiser, W. M. 2005. Nitric oxide emission from tobacco leaves and cell suspensions: Rate limiting factors and evidence for the involvement of mitochondrial electron transport. Plant J. 41:732-743.

Polverari, A., Molesini, B., Pezzotti, M., Buonaurio, R., Marte, M., and Delledonne, M. 2003. Nitric oxide-mediated transcriptional changes in Arabidopsis thaliana.: Mol. Plant-Microbe Interact. 16:1094-1105.

Rumer, S., Kapuganti, J. G., and Kaiser, W. M. 2009. Oxidation of hydroxylamines to NO by plant cells. Plant Signal. Behav. 4:853-855.

Scheler, C., Durner, J., and Astier, J. 2013 Nitric oxide and reactive oxygen species in plant biotic interactions. Curr. Opin. Plant Biol. 16:534-539.

Shi, F. M., and Li, Y. Z. 2008. Verticillium dahliae toxins-induced nitric oxide production in Arabidopsis is major dependent on nitrate reductase. BMP Rep 41:79-85.

Shoresh, M., Harman, G. E., and Mastouri, F. 2010. Induced systemic resistance and plant responses to fungal biocontrol agents. Annu. Rev. Phytopathol. 48:21-43.

Srivastava, N., Gonugunta, V. K., Puli, M. R., and Raghavendra, A. S. 2009. Nitric oxide production occurs downstream of reactive oxygen species in guard cells during stomatal closure induced by chitosan in abaxial epidermis of Pisum sativum. Planta 229:757-765.

Stohr, C., and Ullrich, W. R. 2002. Generation and possible roles of NO in plant roots and their apoplastic space. J. Exp. Bot. 53:2293-2303.

Tun, N. N., Santa-Catarina, C., Begum, T., Silveira, V., Handro, W., Floh, E. I. S., and Scherer, G. F. E. 2006. Polyamines induce rapid biosynthesis of nitric oxide (NO) in Arabidopsis thaliana seedlings. Plant Cell Physiol. 47:346-354.

Turrion-Gomez, J. L., and Benito, E. P. 2011. Flux of nitric oxide between the necrotrophic pathogen Botrytis cinerea and the host plant. Mol. Plant Pathol. 12:606-616.

Viterbo, A., and Horwitz, B. A. 2010. Mycoparasitism. Pages 676-693 in: Cellular and Molecular Biology of Filamentous Fungi. K. A.Borkovich and D. J. Ebbole, eds. American Society for Microbiology, Washington, D.C.

Wang, G. D., Ellendorff, U., Kemp, B., Mansfield, J. W., Forsyth, A. Mitchell, K., Bastas, K., Liu, C. M., Woods-Tor, A., Zipfel, C., de Wit, P. J. G. M., Jones, J. D. G., Tor, M., and Thomma, B. P. H. J. 2008. A genome-wide functional investigation into the roles of receptor-like proteins in Arabidopsis. Plant Physiol. 147:503-517.

Woo, S. L., Scala, F., Ruocco, M., and Lorito, M. 2006. The molecular biology of the interactions between Trichoderma spp., phytopathogenic fungi, and plants. Phytopathol. 96: 181-185.

Yamamoto-Katou, A., Katou, S., Yoshioka, H., Doke, N., and Kawakita, K. 2006. Nitrate reductase is responsible for elicitin-induced nitric oxide production in nicotiana benthamiana. Plant Cell Physiol. 47:726-735.

Yedidia, I., Benhamou, N., and Chet, I. 1999. Induction of defense responses in cucumber plants (Cucumis sativus L.) by the biocontrol agent Trichoderma harzianum. Appl. Environ. Microbiol. 65:10611070 .

Yoshioka, H., Asai, S., Yoshioka, M., and Kobayashi, M. 2009. Molecular mechanisms of generation for nitric oxide and reactive oxygen species, and role of the radical burst in plant immunity. Mol. Cells 28:321-329.

Zeidler, D., Zahringer, U., Gerber, I., Dubery, I., Hartung, T., Bors, W., Hutzler, P., and Durner, J. 2004. Innate immunity in Arabidopsis thaliana: Lipopolysaccharides activate nitric oxide synthase (NOS) and induce defense genes. Proc. Natl. Acad. Sci. U.S.A. 101:15811-15816.

Zhu, Q. H., Stephen, S., Kazan, K., Jin, G. L., Fan, L. J., Taylor, J., Dennis, E. S., Helliwell, C. A., and Wang, M. B. 2013. Characterization of the defense transcriptome responsive to $F$. oxysporum arium oxysporuminfection in Arabidopsis using RNA-seq. Gene 512:259-266. 